\title{
DIFICULTADES DE LOS ESTUDIANTES CON EL PAPEL ESPECÍFICO DEL CAMPO ELÉCTRICO EN LA ÓPTICA
}

\author{
BROSSEAU, C. \\ CERMO. Université Joseph Fourier. BP. 87, 38402 Saint-Martin-d'Hères, Cedex, France.
}

\section{SUMMARY}

This paper deals with the difficulties of the understanding of the specific role of the electric field in Optics among students enrolled in undergraduate physics courses. Wiener's experiment along with symmetry arguments provide the basis for an instructional strategy designed to address this specific difficulty.

\section{INTRODUCCIÓN}

La óptica es ciertamente uno de los temas de física más difíciles de enseñar. La aproximación convencional a la óptica trata principalmente de introducir los efectos de polarización como una consecuencia de la descripción vectorial de la luz a través de la teoría del campo electromagnético de Maxwell. Más concretamente, en general se dice en los libros de texto que las propiedades de polarización de la luz se originan en el vector eléctrico del campo de onda. Hay una impresión generalizada en muchos enseñantes (incluidos nosotros) de que los es. tudiantes a veces se sienten incómodos con esa afirmación y tienen una apreciación inadecuada de qué son las cualidades específicas de las dos fuerzas fundamentales de campo eléctrico y magnético que describen el campo magnético de la luz, aunque puedan tener faciliđad para manipular matemáticamente estos conceptos. Mi interés aquí se centra en introducir esta dificultad conceptual. He estado implicado durante varios años en un estudio sobre la manera en que los estudiantes de primer curso de electromagnética y óptica piensan sobre la polarización. La perspectiva tomada en esta nota refleja la experiencia surgida de esta actividad de investigación. La estructura de esta nota es como sigue: para comenzar la discusión, citaremos varios ejemplos de presentaciones de libros de texto normales que comienzan con la afirmación arriba mencionada al comienzo mismo del libro. Después se da una discusión de algunos aspectos de las maneras en que esta dificultad específica puede ser vencida.

\section{FORMULACIÓN DEL PROBLEMA E IDENTIFICACION DE LAS DIFICULTADES ESPECIFICAS}

Un examen cuidadoso de la exposición estándar de la óptica de polarización revela que muchos libros de texto son bastante vagos en su descripción básica de la luz polarizada. Para comenzar la discusión permítasenos explorar varios ejemplos sobre este tema. En el texto de Crawford (1965) encontramos: "Describimos los estados de polarización por una cantidad vectorial que denota el campo eléctrico transversal $\mathbf{E}$...» $\operatorname{Read}(1980)$ dice: «Una característica importante de las soluciones de la onda es que el campo eléctrico existe sólo en una dirección transversal. Este tipo de onda se describe como polarizado linealmente... Hasta ahora hemos prestado poca atención al campo magnético $\mathrm{H}$ en una onda plana, aparte de percibir que tiene una amplitud proporcional a $\mathbf{E}$...» En una de las exposiciones más disponibles de óptica de polarización, Shurcliff escribe (1966): «Se podría, por supuesto, preferir tratar de la vibración magnética más que de la eléctrica. Cuando la luz viaja en el vacío u otro medio isotrópico, estas dos vibraciones son ortogonales y sus magnitudes son proporcionales entre sí. Especificar una es tanto como especificar ambas. La decisión de concentrarse en la vibración eléctrica es convencional y es un tributo al papel dominante del vector eléctrico en los procesos de absorción.» 
En este y en otros conocidos libros de texto se ha asumido tácitamente que para explicar los efectos de la polarización es suficiente especificar el comportamiento del campo eléctrico $\mathbf{E}$ porque entonces el campo magnético $\mathbf{H}$ puede ser determinado desde $\mathbf{E}$ mediante las ecuaciones de Maxwell. Desde un punto de vista físico normalmente se acepta que no importa de qué vector se trate. Hemos identificado esta tendencia también a través de entrevistas individuales que se han llevado a cabo en los dos últimos años (Brosseau 1992) entre diferentes estudiantes ( $\sim 50$ estudiantes, la mayor parte de ellos de segundo año de universidad: todos estos estudiantes habían asistido a un curso de un semestre de electromagnética y óptica). Unas pocas palabras sobre la metodología que utilizamos. En el curso de las entrevistas se hicieron dos preguntas a los estudiantes: ¿Por qué la luz puede ser polarizada y cuál es la base física en el origen de este efecto? Como Ia luz es un efecto electromagnético, es decir, caracterizado por ambos campos, eléctrico y magnético, ¿conoces argumentos experimentales o teóricos que relacionen la polarización con estos campos? Esta investigación se llevó a cabo principalmente como un estudio descriptivo más que cuantitativo. Sin entrar en gran detalle, del análisis de estas entrevistas surgen dos resultados interesantes. Primero, es instructivo enfatizar que la polarización no tiene un significado compartido comúnmente en la vida cotidiana (por ejemplo, la visión humana noescapaz de detectar la dirección de la polarización de la luz). Los estudiantes generalmente no entendían el concepto lo suficientemente bien como para determinar un procedimiento experimental que pudieran usar en una situación física real o un razonamiento físico formal para probar que existe una fuerte conexión entre la polarización y el campo eléctrico de la luz. Hemos podido identificar el hecho de que el que la ecuación de d'Alembert describa la propagación de ambos campos comobora las concepciones de los estudiantes de que $\mathbf{E}$ y $\mathbf{H}$ son equivalentes en un sentido matemático: nuestra interpretacion de las respuestas en las entrevistas es que, una vez que se han introducido las ecuaciones, existe una tendencia común a evitar pensar de forma crítica sobre la situación física. El problema no podría ser atribuido a dificultades de computo (se vio que los estudiantes con grandes habilidades matemáticas tenían los mismos síntomas) sino más bien a dificultades conceptuales. Así, los estudiantes llegaron a contemplar la polarización como una propiedad eléctrica abstrusa de la luz, una propiedad no sujeta a explicación física y para la que no existe una figura física concreta. Podría añadirse que asimilar $\mathbf{E}$ y $\mathbf{H}$ es también abrir camino a objeciones, ya que, si efectivamente el tratamiento clásico de los fenómenos ópticos puede limitarse a la discusión del vector eléctrico, esto ya no es cierto a nivel cuántico por la inconmutabilidad de los operadores de campos asociados con los campos clásicos (Louisell 1973).

\section{UNA ESTRATEGIA INSTRUCCIONAL PARA ACOMETER ESTAS DIFICULTADES}

Una introducción a la Iuz polarizada podría comenzar con esta pregunta: ¿qué podemos proponer a los estu- diantes para que puedan tener una definición operativa de la polarización y puedan relacionarla con el campo electromagnético? Hay dos ideas que pueden considerarse para contestar esta pregunta: una (a) deriva del experimento directo, mientras que la otra $(b)$ se basa en consideraciones teóricas.

a) La falta de una base experimental concreta en los libros de texto deja un hueco en la comprensión conceptual de la polarización. Esta situación no es satisfactoria, pues es conocido que la instrucción científica es más efectiva cuando una experiencia concreta establece la base para la construcción de conceptos científicos. Rellenar este hueco puede conseguirse refiriéndose al experimento de Wiener. En 1890 Otto Wiener publicó un trabajo en Annalen der Physik (Wiener 1890, 26 años đespués de que Maxwell completase su trabajo) en el que sugería un inteligente experimento para ilustrar el papel del campo eléctrico en la óptica. Desgraciadamente este brillante experimento no ha recibido el amplio reconocimiento que merece: normalmente no es reproducido en los libros de texto de óptica, y en las entrevistas exploratorias encontramos que los estudiantes nunca habían oído hablar de él. Un breve comentario de este experimento puede ayudar en nuestra discusión y pro. cedemos a darlo ahora. Se hacen referencias al trabajo original de Wiener (1890), y a Born y Wolf (1980) para una exposición más detallada.

Un rayo de luz casi monocromática de una longitud de onda central $\lambda$ incide sobre una superficie que refleja totalmente $\Sigma$ (espejo de plata) en el plano $x_{3}=0$ (Fig. 1). Una película fotográfica transparente fina $(<\lambda / 20) \mathrm{F}$ que cubre una placa de cristal $G$ se coloca como se ilustra en la figura 1 con un pequeño ángulo $\delta$ (igual a 4 minutos de arco en el trabajo original de Wiener). El principal resultado del experimento de Wiener es que la película fotográfica aparece ennegrecida en bandas regulares. Sean $\mathrm{E}_{\mathrm{j}(\mathrm{r})}$ y $\mathrm{H}_{\mathrm{i}(r)}$ los campos eléctrico y magnético, los subíndices $i$ y $r$ significando las ondas incidente y reflejada. De los cálculos de la dependencia espacial de los

Figura 1

Iłustración de las notaciones del experimento de Weiner.

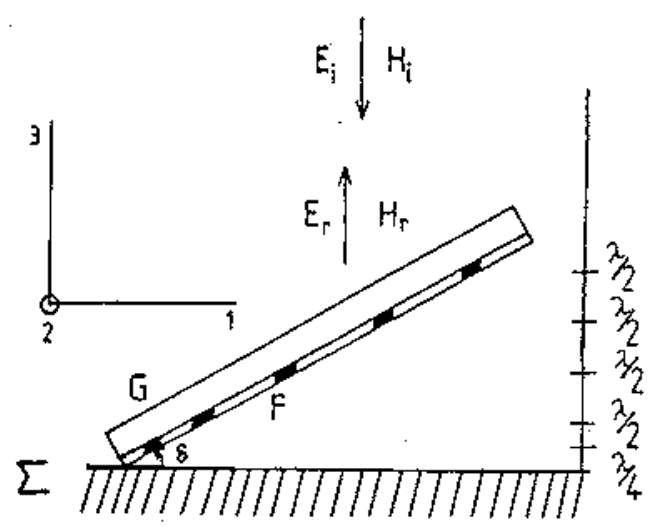


componentes $\left(\mathrm{x}_{1}, \mathrm{x}_{2}\right)$ de las amplitudes totales de vectores de campo, se encuentra que a lo largo de $x_{3}$

$$
\begin{aligned}
& \mathrm{E}_{\mathrm{l}}=\mathrm{E}_{\mathrm{li}}+\mathrm{E}_{\mathrm{lr}} \sim \operatorname{sen}\left(2 \pi \mathrm{x}_{3} / \lambda\right) \\
& \mathrm{H}_{1}=\mathrm{H}_{\mathrm{li}}+\mathrm{H}_{\mathrm{lr}} \sim \cos \left(2 \pi \mathrm{x}_{3} / \lambda\right)
\end{aligned}
$$

con $l=x_{1}, x_{2}$. Las ecuaciones ( $\left.1 \mathrm{a}-\mathrm{b}\right)$ son funciones periódicas de $\mathrm{x}_{3}$ : los nodos (amplitud mínima) de $\mathrm{E}$ vienen dados por $\mathrm{x}_{3 \mathrm{~m}}=\mathrm{m} \lambda / 2$ con $\mathrm{m}=0,1,2 \ldots$ en tanto que los antinodos (amplitud máxima) son $x_{3 \mathrm{~m}}=\mathrm{m} \lambda / 2$ con $\mathrm{m}=1 / 2,3 / 2$, $5 / 2$... Como puede verse, los nodos de $\mathbf{E}$ son los antinodos de H. Dos puntos son también dignos de mención. Primero, el espejo reflectante es un nodo de E. Segundo, en el experimento de Wiener se encontró que el ennegrecimiento de la película ocurría sólo en las posiciones de los antinodos de $\mathbf{E}$. En consecuencia, la acción fotoquímica está directamente relacionada con el vector eléctrico, y no con el magnético. Como Born y Wolf (1980) hicieron notar «esto es de esperar por la teoría del electrón, ya que el proceso fotográfico es un proceso đe ionización en que el electrón es extraído de una unión atómica de haluro de plata, y la fuerza electromagnética sobre una partícula de carga en reposo es proporcional al vector eléctrico". Obsérvese que en lugar de la enulsión fotográfica empleada por Wiener (1890) se usaban otros tipos de detectores tales como películas fluorescentes y películas foto-emisoras: la respuesta máxima se encontró en todos los casos en los antinodos del campo eléctrico (Born 1980). En suma, este experimento proporciona una atractiva figura que establece que la polarización no está asociada con la estructura interna de la luz, sino más bien con la estructura de su campo eléctrico. La mayor parte de las técricas experimentales están relacionadas con el campoe léctrico vía medida de intensidad (observable en frecuencias ópticas que son una función cuadrática de E), y en la mayor parte de la interacción entre la luz y la materia (por ejemplo, el efecto de la luz sobre una emulsión fotográfica, como en el experimento de Wiener), es el vector eléctrico el que está activo.

b) El segundo argumento que es relevante para enfatizar el papel específico del campo eléctrico en la optica se basa en la simetría discreta de inversión de tiempo (simetría-T) (Wigner 1982). Las leyes de física fundamental están sujetas a una cantidad de transformaciones de simetría. Sus propiedades de simetría imponen restricciones sobre las posibles propiedades de materia y campos. Puesto que las ecuaciones de movimiento de la mecánica clásica son ecuaciones diferenciales que implican derivadas temporales de segundo orden, son invariables bajo la transformación de simetría- $T t \rightarrow-t$ mientras que funciones extrañas de $t$, tales como la velocidad v, cambian de signo. Como resultado, surge una diferencia principal entre cantidades estáticas y dinámicas. Así, en el punto $r$, la densidad de carga $(r, t)$ y la actual densidad $j(r, t)$ se comportan de forma diferente bajo simetría-T: $(\mathbf{r}, \mathbf{t}) \rightarrow(\mathbf{r},-\mathbf{t}) ; \mathbf{j}(\mathbf{r}, \mathbf{t}) \rightarrow-\mathbf{j}(\mathbf{r},-\mathbf{t})$.

Consideremos ahora la ecuación de movimiento de una partícula de masa $m$ y carga $q$ moviéndose en el vacío bajo la influencia de la fuerza de Lorentz en un campo eléctrico $\mathbf{E}(r, t)$ y un campo magnético $\mathbf{H}(r, t)$. Esta ecuación es (cuando $|v| / c<<1$ ):

$$
\mathrm{mr}=\mathrm{qE}(\mathrm{r}, \mathrm{t})+\mu_{0} \mathrm{q} v \times \mathbf{H}(\mathrm{r}, \mathrm{t})
$$

$\mu_{0}=1.2610^{6} \mathrm{Hm}^{-1}$ y la diéresis indica la diferenciación de tiempo. Es un hecho experimental que la carga eléctrica es invariable bajo las transformaciones galileanas y es escalar bajo la simetría-T (Jackson 1967). Entonces, de acuerdo con la observación previa, la ecuación 2 será invariable bajo la simetría- $T$ si, y sólo si $\mathbf{E}(\boldsymbol{r}, t) \rightarrow-\mathbf{H}(\mathbf{r},-\mathbf{t})$. Esta conclusión, por supuesto, es de esperar por la teoría de campo electromagnético de Maxwell. El campo eléctrico se produce por una distribución de cargas estáticas $(r, t)$ no afectadas por la simetría-T en tanto que el campo magnético se origina por una distribución de cargas eléctricas en movimiento $\mathrm{j}(\mathrm{r}, \mathrm{t})$ cuyo signo es invertido por la simetría-T. Los campos eléctrico y magnético no aparecen simétricamente en las ecuaciones de Maxwell y Lorentz. Este punto importante fue enfatizado por Rosen (1973) como sigue: «Mientras que existen en la naturaleza cargas eléctricas que sirven como fuentes de campos eléctricos y detectoras de ello cuando en movimiento, sirven también como fuentes de campo magnético y detectoras de ello, no existen, al menos por lo que actualmente se conoce, cargas magnéticas (monopolos magnéticos) que en reposo produzcan y detecten campos magnéticos y en movirmiento también produzcan y detecten campoeléctrico. Si alguna vez se descubrieran tales cargas magnéticas, entonces los campos eléctricos y magnéticos serían completamente simétricos». De la ecuación 2 se podría añadir que la ratio de la magnitud de las fuerzas eléctricas a las magnéticas no puede exceder $|\mathbf{y}| / \mathrm{c}$, siendo $c$ la velocidad de la luz en el vacío. Ahora bien, en el alcance de las frecuencias ópticas, los electrones tienen sólo velocidades pequeñas (Heitler 1984) en un periodo; por tanto, los efectos magnéticos normalmente no son importantes. Una vez se ha determinado la polarización del campo eléctrico, la polarización del campo magnético puede ser caracterizada mediante las ecuaciones de Maxwell.

\section{CONCLUSIÓN}

Los argumentos presentados más arriba tienen una implicación importante para ayudar a los estudiantes a formular una definición operativa de polarización. Parece que los estudiantes que recibieron este material de instrucción tienen menos dificultades de razonamiento en cursos más avanzados de electromagnética y óptica que los que no lo recibieron (Brossean 1992). Otra aplicación es que tal investigación de la comprensión de los estudiantes puede proveer con una fuente de ideas para problemas y experimentos de laboratorio. En resumen, el objetivo de nuestra instrucción es ayudar a los estudiantes: a) a pensar en el enunciado del problema (aquí, descripción de la polarización de la luz) describiendo un proceso físico (aquí, en términos de campos eléctricos y magnéticos); $y(b)$, construir una estructura de conocimiento (visualizar e identificar el papel específico del campo eléctrico) en torno a los conceptos básicos de la física. 
Para concluir, nos referimos a dos citas que hemos encontrado relevantes para nuestro propósito. Citando a Feynman y otros (1970) «Cuando hablo de los campos zumbando a través del espacio, tengo una terrible confusión entre los símbolos que utilizo para describir los objetos y los objetos mismos. Realmente no puedo formarme un cuadro que sea ni tan sólo aproximadamente como las ondas reales... Quizás la única esperanza sea tomar una visión matemática. Desde un punto de vista matemático, hay un vector de campo eléctrico y un vector de campo magnético en cada punto del espacio: es decir, hay seis números asociados con cada punto en el espacio. ¿Puede imaginarse seis números...? Desgraciadamente estamos limitados a abstracciones, a utilizar instrumentos para detectar el campo, a utilizar símbolos matemáticos para describir los campos... Pero, a pesar de todo, en algún sentido los campos son reales, porque después đe haber acabado de jugar con ecuaciones matemáticas, podemos aún hacer que los instrumentos detecten las señales del Mariner II y hacer averiguaciones sobre galaxias que están a billones de millas, y así sucesivamente.» Como McDermott (1991) expresó con tanto acierto: «Los estudiantes deberían adquirir una clara comprensión de los conceptos físicos básicos que pueden definir operativamente y unirlos de forma significativa a los principios importantes.» Estas dos citas resaltan la dificultad de relacionar conceptos físicos y sus representaciones con objetos reales. El desarrollo que hemos hecho antes sugiere que ello es particularmente cierto en la 6 ptica de polarización. Como comentario final, creemos que dedicar más atención de la que actualmente se dedica a los conceptos básicos es importante para comprender y apreciar en toda su extensión el concepto de polarización óptica. En vista de recientes y urgentes demandas para la enseñanza de la óptica (Hall 1991), disipar estas dificultades conceptuales adquiere una nueva urgencia.

\section{REFERENCIAS BIBLIOGRÁFICAS}

BORN, M. y WOLF, E., 1980. Principles of Optics. (Pergamon Press: Oxford).

BROSSEAU, C., 1992. Unpublished.

CRAWFORD, F.S., 1965. Waves, Berkeley Physics Courses, Vol. 3. (McGraw-Hill: Nueva York).

FEYNMAN, R.P., LEIGHTON, R.B. y SANDS, M., 1970. The Feynman Lectures on Physics, Electromagnetism 1. (AddisonWesley: Reading).

HALL, D., 1991. Photonics education-the new challenges, Physics World, Vol. 4, p. 14.

HEITLER, W., 1984. The Quantum Theory of Radiation, 3rd ed. (Dover, Nueva York).

JACKSON,J.D., 1967.Classical Electrodynamics, cap. 7.(Wiley, Nueva York).

LOUISELL, W.H., 1973. Quantum Statistical Properties of Radiation. (Wiley: Nueva York).
MCDERMOTT, L.C., 1991. What we teach and what is learned. Closing the gap, Am. J. Phys., Vol. 59, pp. 301-321.

READ,F.H., 1980. Electromagnetic Radiation, p. 19-22. (Wiley: Chichester).

ROSEN, J., 1973. Transformation Properties of Electromagnetic Quantities under Space inversion, Time reversal and Charge conjugation, Am. J. Phys., Vol. 41, pp. 586-590.

SHURCLIFF, W.A., 1966. Polarized Light. (Harvard Univ. Press: Cambridge).

WIENER, O., 1890. Stehende Lichtwellen und die Schwingungsrichtung polarisirten Lichtes, Ann. d. Physik, Vol. 40, pp. 203-267.

WIGNER, E.P., 1982. Group Theory, cap. 26. (Academic Press: Nueva York). 